\title{
NUTRITION AND MULTIPLE ORGAN FAILURE
}

\author{
KENT LUNDHOLM, ANDERS HYLTANDER AND ROLF SANDSTRÖM \\ Department of Surgery, University of Göteborg, Sahlgrenska Hospital, Göteborg, \\ Sweden
}

\section{CONTENTS}

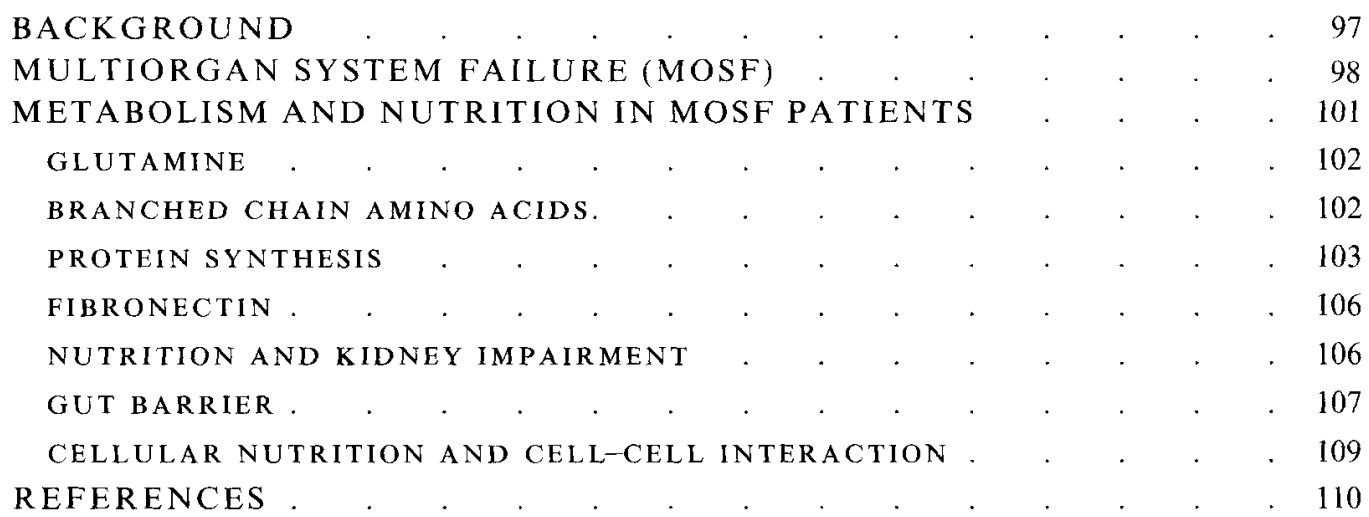

\section{BACKGROUND}

Normal cellular homeostasis is dependent on adequate oxygenation, substrate delivery and excretion of metabolites. The development of modern medicine has experienced a remarkable progress of knowledge in this area over the recent decades. Circulatory and respiratory support modalities for critically ill patients have been developed due both to increased understanding of cellular and organ metabolism and to improved technology. Improvements are dependent on both artificial organs and supportive modalities including drug therapy. Such basal devices provide means by which the cellular supply of oxygen, energy and excretion of metabolites are fulfilled. The progress in nutrition research also represents a giant step forward including decreased morbidity and improved therapeutic outcome in patients suffering from a variety of disorders such as injury, infection with septic complications and eventual multiple organ failure (Baue, 1991; Phelps et al. 1991). For several reasons it has, however, been difficult to confirm or demonstrate the role of nutrition in itself to predict outcome in severely ill patients. There are several reasons for this: the difficulty of conducting controlled studies in patients with life-threatening disorders, the heterogeneity of patients treated in intensive care units, and the ignorance of modern principles in clinical studies with appropriately designed protocols (Friedman et al. 1981).

Intravenous nutrition was developed when it was recognized that patients without early and uncomplicated recovery suffered from progressive starvation leading to the appearance of complications such as increased susceptibility to infection and impaired wound healing. Such observations have since then been repeatedly confirmed by systematic evaluation in 
different kinds of patient with various degrees of undernutrition. It is thus well established that patients suffering from undernutrition have poor outcome and exhibit slower rehabilitation than well-nourished patients (Bistrian et al. 1976). This fact has led to the conclusion and expectation that artificial nutrition, either parenterally or enterally infused, should improve outcome and shorten convalescence. Numerous studies have, however, failed to demonstrate this clearly (Detsky et al. 1987; VA Total Parenteral Nutrition Cooperative Study Group, 1991). It is quite obvious that the supply of nutrients is a prerequisite for survival of patients with injury and organ failure. The question is not what the role of artificial nutrition is in these patients, but how nutrition should be given (the calorie and nitrogen load), the composition of nutrients, and the optimal time course for institution of various nutrition modalities. It has recently been supposed that supranormal provision of non-protein calories and of protein was desirable on the grounds that injured patients had an increased metabolic rate. More recently, it became clear that such supranormal provision of nutrients induced other disturbances and certainly represented a trigger which promoted progress in dysfunctions and an imbalanced homeostasis. A most remarkable example of this was the observation that hypernutrition prolonged the need for ventilation support and even provoked respiratory failure in patients with borderline respiratory function (Quinn \& Askanazi, 1987; Shaw-Delanty et al. 1990). This is in part explained by the thermal effect of nutrients, particularly carbohydrates and amino acids, which leads to increased oxygen consumption and the need for increased removal of carbon dioxide and also stimulation of cellular metabolism induced by substrates and hormones (Askanazi et al. 1981; Greig et al. 1987).

\section{MULTIORGAN SYSTEM FAILURE}

Organ failure due to hypermetabolism continues to be one of the most serious conditions of surgical patients. It has been reported to occur after $10-20 \%$ of emergency operations and in $30-50 \%$ of patients with intra-abdominal abscess (Sturm et al. 1979; Fry et al. 1980). The reported mortality in these patients ranged from 30 to $100 \%$ varying according to the number of organs with malfunction. In patients with three or more organs failing, the mortality is usually close to $100 \%$. Many risk factors have been associated with organ malfunction and death. The causes are multifactorial and several factors are certainly additive and may even be synergic. Sepsis occupies a central role in the sequence of events, and the risks are associated with age, malnutrition, severity and continuance of shock, preexisting disease or limited organ function, although definitions and the clinical variables have not been standardized. In a previous report it was found that shock, age, previous alcohol abuse, intestinal infarction and malnutrition in that order were the factors which explained with highest probability the likelihood of poor outcome and death (Pine et al. 1983). Shock is the most likely cause of death, and shock at any time was associated with a $70 \%$ mortality compared to $5 \%$ in patients who never experienced a shock period, although a certain degree of variability may occur depending on the kinds of patients that are treated and evaluated (Pine et al. 1983). Lung dysfunction is most frequent, and few patients with one or more than two organs involved fail to develop respiratory distress. It appears that the lungs are involved in all patients that die from multiorgan systemic failure (MOSF). Thus, heart, liver and central nervous system (CNS) malfunctions seldom occur in isolation and are uniformly associated with high risk for death. Kidney malfunction occurs in isolation and represents a single organ failure that can lead to death in a significant number of patients, particularly when continuous arteriovenous haemodialysis is not available. Table 1 suggests some criteria which describe MOSF. 
Table 1. Criteria of Multiple Organ Failure as described by Jordan et al. (1987)

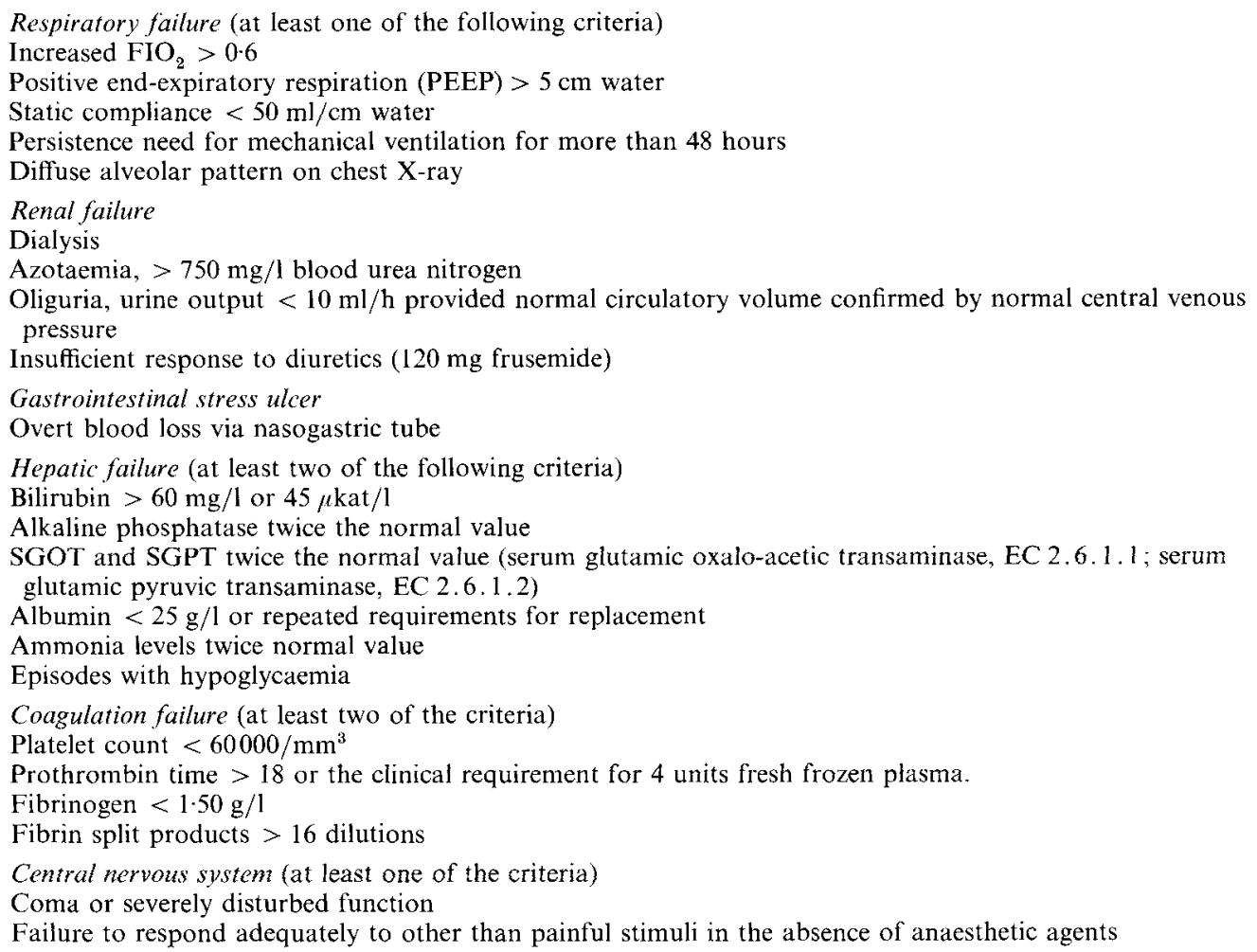

Several studies have described sepsis and its complications as the major cause of mortality in patients in intensive care units (ICU) (Jordan et al. 1987). Patients with MOSF have an identifiable clinical course that can be described and quantified by various scores. The most frequently used is the Acute Physiology and Chronic Health Evaluation assessment (APACHE) that describes a patient's physiological status on admission and grades the deviation from normal of selected organs systems (Knaus et al. 1984). The Therapeutic Intervention Scoring System (TISS) grades the amount of medical and mechanical support needed by the patient, establishes a measure of the therapeutic input needed, and can predict outcome (Keene \& Cullen, 1983). The most valuable use of such scoring systems in the medical literature has been to describe populations under therapeutic evaluation. An additional clinical rating system which assessed septic patient's ongoing disease course and severity has been reported (Jordan et al. 1987). This system incorporates the TISS and APACHE scores to measure discrete organ system abnormalities plus a scale which quantifies a number of abnormal organ systems (Jordan et al. 1987). The resulting score reflecting the severity of dysfunction and grading the responsiveness to therapy was validated against actual disease courses, and it was suggested that the method was more effective in indicating severity of sepsis and more sensitive in monitoring day-to-day changes in patient's clinical status than previous techniques.

Infection is obviously a key factor initiating and promoting MOSF in surgical patients, although haemorrhage and tissue destruction may be initial events. Subsequent bacteraemia 
Table 2. Examples of infection criteria and associated physiological abnormalities in patients with multisystem organ failure, as reproduced from Jordan et al. (1987). The patient must meet two of the six criteria

\section{Hypotension}

Blood pressure remaining below $80 \mathrm{mmHg}$ for $2 \mathrm{~h}$ without haemorrhage or cardiac ischaemia. The patient must meet all of the following criteria:

Tachycardia $>110$ beat $/ \mathrm{min}$

Cardiac index $>31 / \mathrm{min}$ per min per $\mathrm{m}^{2}$

Central venous pressure $>12 \mathrm{mmHg}$

Pulmonary capillary wedge pressure $>16 \mathrm{mmHg}$

Systemic vascular resistance $<600$ dyn sec $/ \mathrm{cm}^{2}$

Infection-positive cultures from any of the following

Blood, sputum, urine, wound, abdominal abscess, peritoneal fluid

Haematological abnormalities

Prothrombin time $>18 \mathrm{~s}$

Platelet count $<90000 / \mathrm{mm}^{3}$

White blood cell count $>15000$ or $<2000 / \mathrm{mm}^{3}$

Respiratory

Positive chest X-ray for pneumonia, X-ray confirmed abdominal air in the absence of previous surgery

Metabolic abnomality

Acidosis and increased anionic gap

and endotoxinaemia develop due to impaired integrated physiological structures in a variety of tissues such as skin, oropharynx, gut and the lungs. A number of experimental studies have demonstrated that most of the bacteria found in the blood after shock were enteric organisms, suggesting that they originated from the gastrointestinal tract, which has been regarded as a reservoir of bacteria and endotoxins, and the gut may become leaky for various reasons following stress (Van Saene et al. 1983; Marshall et al. 1987). By feeding labelled Escherichia coli to animals, it has been demonstrated that the radiolabelled bacteria escape from the gastrointestinal tract during shock and appear in the blood stream. This kind of experiment demonstrates a link between trauma and infection that might not be obvious in all circumstances (Rush et al. 1988). Moreover, it may be most difficult to recognize, since positive blood cultures are lacking in a great number of patients, particularly in those with most severe symptoms of distress and insufficient circulation. Negative blood cultures were found in about $3 / 4$ of all patients suffering unstable circulation following trauma, with most positive blood cultures in patients with blood pressure lower than $80 \mathrm{mmHg}$ at admission. Both Gram-negative and Gram-positive bacterial specimens were found (Rush et al. 1988). In some patients without living bacteria, endotoxins were confirmed. Thus, burn and trauma among other conditions may lead to the translocation of bacteria and endotoxin from the gut or outside epithelial linings to local lymph nodes and sometimes to other organs such as the liver and the lungs. These events are probably based on hyperpermeable membranes, which also include capillaries and membranes in the aerodigestive tract (Offenbartl \& Bengmark, 1990; Steinmetz \& Meakins 1991). It is thus likely that early bacteraemia combined with a simultaneous downregulation of the immune system, perhaps a part of negative feedback mechanisms on immunity, may set the stage for sepsis and multiorgan failure seen in ICU. Like burned patients and patients with severe acute pancreatitis, patients with acute severe trauma and haemorrhagic shock are subject to a two-phase process. In the first phase there is extracellular fluid depletion, perhaps accompanied by membrane hyperpermeability and bacteraemia. These events lead to the second phase, which is the septic state; it is this phase 
with its accompanying multiple organ failure that constitutes the irreversible shock syndrome.

An alternative to endogenous bacteria may be nosocomial infections in injured patients. Nosocomial infections may include contamination of the skin, mucosal membranes, or the wound. In such environments, bacteria may start to multiply to high concentrations including colonization, bacterial resistance and a decreased host capacity to resist colonization/infection with aerobic bacteria. This characteristic may be more linked to the host than to the micro-organism. Sepsis is thus the systemic inflammatory response of the host to an invading micro-organism or corresponding toxin. This response is generally a host dependent function and is necessarily not linked to the invading organism. Other agents that can activate the systemic inflammatory response are necrotic tissue, haematoma and micronecrosis due to microcirculatory flow impairment after perfusion failures. This fact explains the low percentage of positive blood cultures, less than $15 \%$, in clinically septic patients with MOSF (Offenbartl \& Bengmark, 1990). Table 2 describes the relationship between infection and physiological abnormalities in patients with MOSF.

\section{METABOLISM AND NUTRITION IN MOSF PATIENTS}

Current nutrition support regimens can control specific nutrient deficiencies and prevent starvation becoming a morbidity or mortality factor (Müller et al. 1982). However, it has been difficult to demonstrate that nutrition in itself is a major factor that reduces mortality and improves outcome (Detsky et al. 1987; VA Total Parenteral Nutrition Cooperative Study Group 1991). Although the hypermetabolic organ failure complex has had a progressive reduction in mortality risk from $90 \%$ to levels around $35-40 \%$ the underlying mechanisms are still hypothetical. The metabolic response to trauma and surgery is maximal on days $2-4$ and usually abates on days $7-10$. In a subgroup of patients hypermetabolism persists or returns after a period in a reduced form followed by signs of multiple organ failure (Cerra, 1987). The transition from the normal course is generally manifested by the appearance of a lung injury. Sometimes the lung injury is the dominant feature with organ failure being a preterminal feature. Alternatively, lung, liver and renal dysfunction present at an early stage: a stable phase of 7-10 days is entered and then progressive deterioration occurs. The phase of persistent hypermetabolism is characterized by high oxygen consumption, nitrogen excretion in excess, and increased lactate production in peripheral tissues in a balanced relationship to pyruvate. Amino acids are released from the periphery, including increased output of alanine, glutamine and 3-methylhistidine concomitant with increased blood flow and cardiac output and low vascular resistance (Cerra, 1987). The hormonal alterations are securing availability of substrates, i.e. an increased adrenergic tone, increased glucagon/insulin ratio, hypercortisolism and subsequent down-regulation of thyroid hormones. All these changes provide various organ systems with preferential substrates and nutrients as well as focusing cellular events to optimize survival in a threatening condition. The metabolic alterations include insulin resistance in peripheral tissues and continued gluconeogenesis in the presence of high plasma insulin. Glucose can thus be directed towards tissues that are exclusively dependent on glucose for increased energy consumption, such as brain tissue, red blood cells, some cells in the adrenals and a majority of immune cells, while liver and heart cells can rely more on oxidation of fatty acids, pyruvate and even lactate. Noteworthy is the appropriate oxidation of branched chain amino acids at increased rate in skeletal muscles, that circumvents an insulin dependent energy uptake (Pittiruti et al. 1985). This switch is particularly evident in the presence of an exogenous supply of amino acids to increase otherwise moderately elevated circulating levels. 


\section{GLUTAMINE}

The supply of amino acids for oxidation in the skeletal muscles is otherwise provided by degradation of myofibrillar proteins. This is evident by the increased release of 3-methylhistidine from the skeletal muscles in infected patients concomitant with the production of alanine and glutamine (Sjölin et al. 1990). Alanine is synthesized by amination of pyruvate and glutamine is produced by amination of $\alpha$-ketoglutarate. Thus, a substantial production of alanine and glutamine can occur, although declining intracellular concentrations of glutamine have been interpreted to indicate a shortage of this compound in stressed patients (Sandstedt et al. 1985; Fong et al. 1990). Decreased concentrations of glutamine in injured and septic patients have indicated that extra supply of glutamine to such patients may be fruitful (Stehle et al. 1989; Newsholme, 1990; Smith \& Wilmore, 1990). However, the majority of patients with decreased or low muscular glutamine concentrations produce glutamine in normal or even increased amounts when compared to control patients with similar metabolic rate and activity (own unpublished results). This may be due to increased oxidation of the branched chain amino acids, which possibly represent an endogenous glutamine source (Skeie et al. 1990). So far, it has not been demonstrated that the peripheral production of glutamine is a limiting factor either for alanine production from glutamine in the splanchnic region or for providing a limited metabolic substrate for the enterocytes or immune cells, although a decline in muscle glutamine concentration occurs regularly in stressed patients. It has been inferred that glutamine represents the major substrate for enterocytes, particularly from cell culture experiments. However, it must then be emphasized that few cells can be cultured in vitro without the addition of glutamine, this amino acid being the most important precursor for nucleic acid synthesis and less as an oxidative substrate. In vitro, glutamine becomes an obligatory precursor for nucleic acid synthesis in dividing cells, since the salvage pathway for nucleotide provision is not present in a test-tube experiment. These arguments are valid in most cell culture experiments. It may also be inferred that the major part of glutamine uptake in the splanchnic area is converted to alanine leaving the amino group as an excretory product, as confirmed in normal individuals (Felig, 1975). Evaluated critically, few examples exist from studies on pathophysiological states to support the view that glutamine has a role in critically ill patients other than as a transport vehicle for a toxic nitrogen compound (ammonia) and as an intermediate in the vital acid-base regulation among organs. Based on this view it is not surprising to find that a correlation between glutamine and overall protein synthesis can be demonstrated during certain experimental conditions (MacLennan et al. 1987; Jepson et al. 1988), and that restitution of intracellular glutamine does not necessarily lead to a resumption of normal protein synthesis (Wusteman \& Elia, 1991). In spite of this conservative view on glutamine function in critically ill patients, a large body of information has been provided to suggest glutamine as a key nutrient. However, a common denominator of such experiments is that the animal experimental manipulation has been rather extreme, such as the combination of infection, anorexia and irradiation (Souba et al. 1985, 1990). In other experiments an exclusive role of glutamine has not been confirmed (Barber et al. 1990). Future clinical experiments may illuminate these questions and the discrepancies among experimental data and clinical observations.

\section{BRANCHED CHAIN AMINO ACIDS}

Another controversial and interesting question may be the provision of branched chain amino acids (BCAA) to critically ill patients (Cerra et al. 1983; Skeie et al. 1990; von Meyenfeldt et al. 1990). Numerous studies have been reported on this topic, but most 
protocols have evaluated nitrogen balance and other variables related to protein synthesis. This was logical since the branched chain amino acids were reported to have specific characteristics in stimulating protein synthesis in animal muscles as also confirmed by ourselves in human tissue (Lundholm et al. 1981). So far, results from clinical studies have not seemed to be particularly rewarding compared to conventional amino acid nutrition (von Meyenfeldt et al. 1990). However, it may theoretically be possible to improve nutritional regimens for severely ill patients by increasing the provision of BCAA as a fuel substrate, particularly in those patients with signs of severe insulin resistance (Black $e t a l$. 1982; Brooks et al. 1986). It is a frequent observation that BCAA are taken up in skeletal muscles after an overnight fast, even in the presence of a net release of the majority of other amino acids (Möller-Loswick et al. 1991). Since this uptake is not associated with corresponding changes in tissue pool sizes, it must imply oxidation (Lundholm et al. 1987; Arfvidsson et al. 1991). Although not systematically evaluated in a large series of patients, it seems as if this uptake is exaggerated in traumatized patients (Pittiruti et al. 1985). Patients with MOSF have also been reported to sustain increased whole body uptake of branched chain amino acids, particularly of leucine, even late in the disease (Pittiruti et al. 1985). It is likely that a majority of the leucine fraction is utilized in the skeletal muscles as fuel, perhaps also illustrated by an increased plasma clearance of a majority of amino acids in critically ill patients with emerging MOSF. A decreasing clearance of amino acids has also been indicative of a poor prognosis suggesting irreversible hepatic cellular failure (Clowes et al. 1980).

\section{PROTEIN SYNTHESIS}

Findings of increased amino acid uptake in severely ill patients have been interpreted to indicate an increased overall hepatic protein synthesis in such patients explained by the acute phase reaction (Sganga et al. 1985). Our own experiments have pointed to the possibility that indices of hepatic protein synthesis may be increased even in the presence of reduced oxygen uptake in liver cells under certain stress conditions (Ternell et al. 1983). This unexpected observation made us evaluate hepatic protein synthesis by two entirely independent methods in animals with various conditions of stress such as cancer, sterile abscess, and bacterial antigen exposure (Ternell et al. 1988). The results demonstrated that conventional kinetic methods, measuring protein synthesis by amino acid incorporation, indicated an increased rate of hepatic protein synthesis, while density gradient centrifugation of polysomes indicated depressed liver synthesis (Lundholm et al. 1978). These unexpected and discrepant findings suggest that protein synthesis may be a more complicated concept than anticipated, i.e. rates of overall protein synthesis are perhaps only relevant in conjunction with evaluation of the energy cost of protein synthesis. In such aspects it may be relevant to talk about the effect of nutrition on overall protein synthesis. However, the variation in synthesis rate among defined cellular proteins is so large in each condition that overall protein synthesis rates may not provide meaningful information. For example, in the skeletal muscles we have data to support the conclusion that a large number of proteins are maintained and even increased in synthesis during complete starvation, while the overall synthesis of proteins was depressed according to conventional findings (unpublished). In the light of such facts it may be irrelevant to consider providing amino acids and non-protein calories to stimulate protein synthesis in patients. A more appropriate view may be to consider the availability of amino acids and substrates as nonlimiting factors in combination with hormonal and growth factor based regulation of a more defined cellular protein synthesis. The relevance of this suggestion is also supported by the finding that a wound can heal even in the presence of severe negative nitrogen and 
energy balance in critically ill patients. With regard to the liver and its demand for nutrients in patients with MOSF very little is actually known beyond some descriptive findings. Based on normal regulation of liver metabolism, protein synthesis and substrate utilization, it is reasonable to suspect that liver cells would be able to maintain energy homeostasis as long as plasma substrate levels do not consistently fall below fasting levels, which represent the set point where liver cells must be able to fulfil normal intrinsic energy production as well as gluconeogenesis.

Except for albumin, it has not been demonstrated in vivo that liver proteins are regulated by substrate availability to any large extent (Rothschild et al. 1975). Therefore, for critically ill patients it is probably sufficient to provide a mixed nutrition corresponding to, or slightly above, the resting needs of the patients (Abbott et al. 1983). According to this view, it is a key question whether basal needs should be calculated from elevated energy expenditure, or whether the values for premorbid expenditure should be used. Oxygen uptake in critically ill patients is elevated in several organs, but changes from patient to patient and from day to day in individuals may be substantial (Bartlett et al. 1982), and the quoted literature claims a 20-100\% increase (Hunter et al. 1988). Burned patients have in general large increases, but septic patients may occasionally show either high or moderately high values (Dahn et al. 1987). What extremely high values depend on is so far unknown, but a contribution from shivering thermogenesis may be an explanation in septic patients with 40-60\% increased metabolic rate. Shivering thermogenesis may occur even though involvement of the muscular component may not be clinically overt. Increased muscular tension may be enough to increase energy expenditure to a substantial degree. From a metabolic and physiological point of view it may be concluded that resting cellular metabolic rate should not be able to increase beyond $25-30 \%$ in a thermoneutral environment. This elevation corresponds to the highest tolerable increase in body temperature, considering that each degree Celsius in body temperature corresponds to a $10 \%$ increase in metabolic rate. This relationship is valid for a variety of conditions including pharmacological cytokine stimulation in patients (P. Naredi et al. submitted for publication). It is thus a very interesting question whether elevated oxygen consumption reflects a truly increased demand at the cellular level or whether it is rather a matter of badly tuned control of energy expenditure. It may well be that a variety of cells in a certain organ are hypoxic due to previous derangements either in cellular membranes or in capillary endothelial cells. This may create a feedback signal to increase the delivery of oxygen by decreasing the peripheral vascular resistance and increasing cardiac output. Nutrient delivery is then indiscriminately increased by flow dependent mechanisms, and may secondarily stimulate cellular metabolism by substrate-induced mechanisms and catecholamine activation.

Substrate dependent mechanisms are particularly relevant to energy yielding mechanisms, which is illustrated in part by the thermic effect of food in living organisms (Nacht et al. 1987). This effect may be $10-25 \%$ of the basal metabolic rate depending on the physiological state and the means by which nutrition is delivered. Provision of intravenous nutrition covering $160 \%$ of basal energy expenditure in healthy cholecystectomized patients increased their energy expenditure by about $25 \%$, which represents a metabolic burden corresponding to a fulminating sepsis (Hyltander et al. 1992). Thus, when large amounts of nutrition are delivered, this may represent a substantial metabolic burden to patients. In those patients whose circulatory and respiratory systems are borderline it may be a critical factor that may jeopardize the outcome. In a recent study, we randomized patients who underwent major surgical procedures to receive postoperatively either complete intravenous nutrition or glucose/electrolytes only in the postoperative period. (R. Sandström et al. submitted for publication). The results demonstrated that 
inappropriate nutrition was associated with extremely poor outcome. Under- and overnutrition were associated with 21 and $36 \%$ mortality respectively, which are unexpectedly high. In a great number of patients it was noticed that conventional nutrition, with non-protein calories provided at $120 \%$ of resting needs and nitrogen at $0.2 \mathrm{~g} \mathrm{~N} / \mathrm{kg}$ daily, may have been excessive. This conclusion was based on observations that resting energy expenditure and heart rate were significantly higher after an overnight fast in such patients even late in the postoperative period, pointing to the possibility that even 'normal' provision of nutrients may be too high in a challenged organism. If these observations and conclusions are representative of a more general situation in critically ill patients, it is probably unwarranted even to cover basal energy and nitrogen needs in patients with incipient or overt MOSF.

In a recent retrospective study it was found that the mortality was $64 \%$ in ICU patients receiving total parenteral nutrition (TPN), which was more than twice the mortality among ICU patients in general (Chang et al. 1989). This may of course underline the fact that the most severely ill patients received TPN, but it cannot be excluded that TPN precipitated increased morbidity and mortality. These observations raise the question whether it would be of benefit to attenuate hypermetabolism by pharmacological means in patients with MOSF. Adrenergic blockade by phentolamine has been reported to be of help in the treatment of haemorrhagic shock (Grould, 1970), as well as the institution of calcium channel blockers. These positive effects have been ascribed to a relief of $\alpha$-adrenergic constriction particularly at the venous end of capillaries, thereby promoting venous return and improving microcirculation in various shock conditions. Experiments on the effect of adrenergic blockade in experimental multiple organ failure have also been reported (Xianjun et al. 1988). Improvements in pulmonary, hepatic and renal function were observed in phentolamine treated rats suffering from shock and hypoxia. These effects were considered to depend on improved microcirculation. In addition phentolamine administration normalized cellular levels of cAMP in various organs such as liver and lungs. This provides evidence for a direct modulation of cellular function via adrenergic mechanisms in MOSF. Thus, it appears reasonable to suggest that hypermetabolism in MOSF represents a feedback loop which is elevated due to hampered microcirculation or compromised capillary - cellular exchange. An exaggerated substrate load may then worsen the situation and increase cellular damage. A more clinically related phenomenon of overnutrition is the well recognized observation that weaning a patient from the ventilator may require reductions in carbon dioxide production (Hunker et al. 1980). This may be achieved by either lowering the total caloric intake or substituting fat for carbohydrates (Bartlett et al. 1982). However, provision of long chain triglycerides (LCT) in large amounts may increase the metabolic burden by compromising cell function in the liver due to increased lipogenesis and lipid accumulation in the reticulo-endothelial system (RES), which may already be highly activated by haemoglobin degradation and engulfment of endotoxin and bacteria in traumatized patients (Cerra et al. 1989).

In conclusion, patients with MOSF should probably not receive non-protein calories above resting need considering their premorbid body composition, even if their measured resting metabolism is higher. Non-protein energy may be provided as $60 \%$ glucose and $40 \%$ fat. Urine should be regularly checked for the occurrence of kidney glucose spill-over, and glucose provision must be reduced accordingly. Extra insulin should not be used to overcome the well-recognized insulin resistance, but it is used in patients with diabetes mellitus. 


\section{FIBRONECTIN}

Fibronectin, which is a protein synthesized in a variety of cells, is an important opsonin in the defence against intruding organisms and for clearance of foreign structures from the circulation and injured tissue (Saba et al. 1983). Fibronectin was early recognized as a sensitive marker of malnutrition in both animals and man, probably dependent on its short half-life. Decreasing plasma concentrations of fibronectin in traumatized patients correlated with increased susceptibility to septic complications, and most interestingly it was demonstrated that transfusions with cryoprecipitated fibronectin from plasma were associated with circulatory and respiratory improvements in septic patients with MOSF (Saba et al. 1978). These initial studies were later confirmed by others, but unfortunately the observations have not been reconfirmed in prospectively controlled investigations on patients with MOSF. If so, this would be a most significant therapeutic advance, since no single treatment factor except artificial organ support has been found to improve established MOSF in unselected surgical patients, not even continuous antibiotic therapy. However, it was recently reported that provision of purified fibronectin to severely malnourished children improved survival in a blind randomized study (Sandberg et al. 1990).

\section{NUTRITION AND KIDNEY IMPAIRMENT}

The use of intermittent haemodialysis as renal replacement therapy has reduced the mortality rate of acute renal failure (ARF) from nearly $100 \%$ to around $60-70 \%$, although dialysis introduces its own problems. Continuous arteriovenous haemofiltration (CAVH) is an alternative method of renal replacement therapy in which extracellular fluid is continuously ultrafiltered from blood and replaced with intravenous fluid. CAVH allows nutrients to be added to the replacement regime, no longer limited by fear of fluid overload. CAVH does not induce hypermetabolism and haemodynamic instability and offers control over fluids, electrolytes and nutrition. Several reports demonstrate that full nutrition supply is possible in oliguric patients with $\mathrm{CAVH}$. Bartlett and associates reported early that mortality in patients with ARF was significantly correlated with a cumulated negative energy balance when compared to patients with vigorous and early nutritional treatment leading to positive cumulated balances (Bartlett et al. 1986). It was concluded that poor nutrition was a limiting factor in itself, since patients grouped with and without appropriate nutrition suffered from similar critical illness. The difference in energy balance between the two groups was about $700-800 \mathrm{kcal} / \mathrm{day}$, and was statistically significant. Similar observations have been reported by the same authors in their earlier work, although a causal relationship was not then inferred. Extensive use of the CAVH technique has further improved survival in patients with MOSF including ARF. Weiss et al. (1989) reported $50-55 \%$ survival in more than 100 consecutively treated patients between 1982 and 1985 with MOSF and ARF. However, the calorie input for patients who survived was $2060 \pm 740 \mathrm{kcal} /$ day and for those who died $2240 \pm 690 \mathrm{kcal} /$ day. Therefore, it remains uncertain to what extent energy and protein intake predicts outcome in severely ill patients with kidney failure. It cannot be excluded that significant attention to the importance of adequate nutrition was a reflection of real differences in the clinical conditions of the patients rather than the importance of the nutritional support in itself. Only prospective and randomized studies can resolve this dilemma. Recently it was shown that the CAVH technique may be utilized to deliver nutrients to patients during haemofiltration (Roberts et al. 1989). 


\section{GUT BARRIER}

It has been repeatedly emphasized that the trigger to systemic MOSF is bacteria and endotoxins whose effects are mediated by lymphokines and cytokines, and that the main source of endotoxin and bacteria is the gut (Steinmetz \& Meakins, 1991). The idea that the contents of the gut could pose a threat to the individual is not new (Fine et al. 1959; Mutirangura et al. 1989). The gastrointestinal tract contains the largest collection of bacteria and toxins in the body. Several gastrointestinal factors contribute to maintaining the structural and functional barrier against this threat. The barrier system consists of chemical and enzymic processes, mucins secreted throughout the gut, rapid enterocyte proliferation and their subsequent desquamation, immune cells located within the gut wall as well as mucous surface antibodies of the IgA type and the macrophage system lining up in the portal capillary bed within the liver. It has been reported that the character of the small bowel flora can affect the systemic immune response (Marshall et al. 1987, 1988). The delayed-type hypersensitivity response to subcutaneously injected antigens was depressed by colonizing the small bowel with Gram-negative bacteria. In other experiments Kupffer cells in coculture with hepatocytes that were exposed to bacterial endotoxin inhibited hepatic protein synthesis (Cerra et al. 1989). Kupffer cells may act by producing mediators that influence the immune response to antigens originating from the gut, i.e. prostaglandins particularly of the type $\mathrm{PGE}_{2}$. Thus, impairment of the gut barrier may result in uncontrolled and continuous release of antigens and overstimulation of macrophages. A large body of information shows that bacterial translocation and endotoxaemia of gut origin exist in experimental animals (Steinmetz \& Meakins, 1991). There are several mechanisms by which malnutrition may contribute to bacterial gut translocation. Most appealing are the observations that starvation and gut rest rapidly leads to structural alterations and atrophy of the mucosal lining, but starvation may also increase mucosal permeability as does shock (Deitch et al. 1990). However, it is still uncertain to what extent the phenomenon of gut translocation occurs in man. Bacteria have been isolated from mesenteric lymph nodes of patients who have undergone laparotomy due to small bowel obstruction (Deitch, 1989), Crohn's disease (Ambrose et al. 1984) and colorectal cancer (Vincent et al. 1988). Endotoxin has been found in the portal blood of patients with obstructive jaundice (Pain \& Bailey, 1986), and bacteraemia of enteric origin is probably a leading cause of death in leukopenic patients after chemotherapy (Tancrede \& Andremont, 1985). It appears unlikely that severe malnutrition alone would be sufficient to precipitate bacterial translocation, but its combination with other stress factors, particularly episodes with hypoperfusion, may well create the risk. Parenterally fed animals also show impaired epithelial cell proliferation as well as other biochemical and immune alterations in the gastrointestinal tract. Therefore, it may not be just a question of nutrient and substrate availability. It appears that enteral stimulation or the presence of nutrients within the gut lumen is essential for maintaining the gut barrier and the immune competence. This conclusion leads to the question as to how the gut can be protected in injured patients at risk of MOSF. The lack of convenient means to monitor and judge effectively the state of gut homeostasis is a great problem in the clinical setting. Signs of functional ileus and diarrhoea, and laboratory measurements such as bilirubin and liver enzymes, are late and unspecific measures. Therefore, prophylaxis remains the main precaution to decrease the risk of gut barrier dysfunction. Alkalinization by antacid or $\mathrm{H}_{2}$-receptor blockade are effective means of reducing the serious risk of gastric bleeding in stressed patients, but the alkaline milieu may contribute to alteration of the gut microflora leading to colonization of the ventricle with bacteria from the oropharynx. An alternative to gastric alkalinization may be cytoprotection with sucralfate as suggested in clinical investigations (Driks et al. 
1987). A liberal use of broad spectrum antibiotics may result in overgrowth of resistant and pathogenic bacteria. Regimens with both oral and parenteral antibiotics lowered septic morbidity after severe burns (Jarrett et al. 1978) and reduced bacterial peritonitis in patients with varicose bleeding due to liver cirrhosis (Ledingham et al. 1988). However, selective decontamination in an unselected surgical population has been less rewarding.

Recently Border and colleagues reported a retrospective multifactorial analysis of the association between support elements (ventilator days, enteral protein nutrition, antibiotic treatment) and organ insufficiency to explain the variance in the magnitude of septic state in 66 patients with blunt multiple trauma (Border et al. 1987). The magnitude of sepsis correlated positively as expected with factors such as positive endexpiratory pressure, plasma creatinine, the alveolo-arterial oxygen difference, plasma bilirubin etc. All these well-recognized variables in patients with MOSF correlated positively to bad outcome, except enteral feeding by proteins which showed a significant but negative correlation to mortality in all ICU conditions. This was interpreted to suggest that enteral nutrition was an identified factor that in itself could positively influence outcome. Thus, the absence of enteral protein intake was associated with progressively more septic behaviour by all criteria, and this was not compensated for by increased intravenous protein support, which provided almost twice as much protein. All patients who died of sepsis had no enteral intake of protein and were in the enforced supine position. The authors concluded that death from sepsis occurred from blood entry of gut endotoxin, following liver damage and a reduced bile salt secretion so that the endotoxin in the gut became more toxic. It was suggested that oral intake of at least $0.3 \mathrm{~g}$ protein $/ \mathrm{kg}$ daily would lower the risk of septic and fatal complications. Alexander and associates have also reported that in severely burned children an increase in enteral protein intake of $25-50 \%$ relative to recommended intake was associated with considerable reductions in mortality rate, in septic behaviour as judged by antibiotic days, and in the use of erythrocyte transfusion and plasma (Alexander et al. 1980). They have also shown in burned guinea-pigs that enteral protein intake relative to intravenous produced a thicker gut mucosa in combination with an attenuated neuroendocrine response (Mochizuki et al. 1984). Subsequently they reported that intact protein was more effective than the equivalent amount of amino acids (Trocki et al. 1986). Antonacci et al. reported similar findings in severely burned adults and made the same observation of reduced mortality in the high protein group (Antonacci et al. 1984). However, it was not nitrogen balance that was improved, but several aspects of immune function. Seen together, there may be a special effect of enteral protein in reducing the septic magnitude in a way that is not achieved by the intravenous route of administration. If so, the beneficial effects of enteral proteins may be multifactorial, such as stimulation of gut motility, improved enterocyte proliferation, mucin production, bile salt secretion and toxin binding (Steinmetz et al. 1991). Several of these factors may not be related to traditional nutrition regarding energy and amino acid supply. However, from previous work it cannot be excluded that enteral nutrition was preferentially tolerated in the least affected patients (Border et al. 1987). In support of this suspicion may be the finding in a randomized study by Cerra et al. (1988) that enteral nutrition did not prevent multiple organ failure after sepsis with attendant hypermetabolism. There was no reduction in either the incidence of MOSF or mortality attributable to the route of administration. The parenteral group tended to have better visceral protein support; the enteral group had more gut complications such as vomiting, diarrhoea and bloating and were longer in achieving adequate nutrition as well as more variance in input and outcome variables. The authors concluded that the route of nutrition administration did not seem to affect the incidence of postseptic MOSF when hypermetabolism is already present. However, in a third study based on randomization it was found that immediate enteral feeding after abdominal 
trauma significantly reduced sepsis (26 vs. $4 \%$ ) (Moore \& Jones, 1986). In addition, it has been reported that supplementation of standard TPN solutions with $2 \%$ glutamine results in enhanced cellularity of both small bowel and colonic mucosa (Hwanu et al. 1986; Jacobs et al. 1988), s-IgA levels were also higher and translocation rates were lower (Burke et al. 1989). The effect of glutamine supplementation in humans and its clinical relevance in the treatment of severely ill patients remains, however, to be established.

\section{CELLULAR NUTRITION AND CELL-CELL INTERACTION}

In George Blackburn's laboratory considerable work has been performed with animal models of trauma, transplantation and sepsis to reproduce the cascade of responses leading to multiple organ failure. Results suggest that hypermetabolism and septic reactions can be attenuated in the early course of multiple organ failure. This research has suggested that the cellular immune system may represent both a positive and negative modulator for survival in critical illness, and that damaged endothelium is a hallmark of progressive deterioration to organ failure (Bagley et al. 1991). These two concepts may be complementary and attempts are being made to identify steps in the processes that can be prevented or attenuated by cellular nutritional support. In response to thrombin, platelet activating factor, bradykinin, hypoxia and mechanical stress the endothelium can modulate the vascular tone by releasing vasorelaxing factors such as endothelium-derived relaxing factor, prostacyclin and various constrictors such as endothelin. Endothelial damage may therefore result from an imbalance between dilators and constrictors, which is theoretically tractable by nutritional manipulation. Such means are represented by eicosanoids of the 2-series prostaglandins $\left(\mathrm{PGE}_{2}, \mathrm{PGI}_{2}, \mathrm{TXA}_{2}\right)$, and the 4-series leukotrienes $\left(\mathrm{LTB}_{4}, \mathrm{LTD}_{4}\right.$, $\left.\mathrm{LTE}_{4}\right)$, potent inflammatory mediators synthesized from arachidonic acid $(20: 4 \mathrm{n}-6)$, an n- 6 polyunsaturated fatty acid derived from linoleic acid. The LCT used in parenteral and enteral nutrition are from soya-bean and safflower oils, which contain linolenic acid. The use of alternative lipids in nutrition may thus provide a future means to alter the arachidonic derived metabolites, in addition to pharmacological intervention. In this respect, it is of particular interest that the biological effect of fish oils rich in n-3 fatty acids does not appear to be limited to their incorporation into cell membranes. Hence, the incorporation of fish oil into a labile pool by low dose infusion may be as effective as long term administration in manipulating eicosanoid synthesis. In line with these basal observations, Blackburn and colleagues have reported that parenteral administration of fish oil to experimental animals with either burns or endotoxaemia improved energy expenditure, nitrogen balance and metabolic responses (Trocki et al. 1986; Pomposelli et al. 1989). Thus, fish oil may decrease the inflammatory response at many levels. Another stratagem for the manipulation of eicosanoid synthesis may be the use of medium chain triglycerides (MCT). Compared to LCT fed animals, rats fed a diet enriched with coconut oil exhibited a diminished acute phase response to endotoxin stimuli (Wan \& Grimble, 1987). MCT may also be associated with a more rapid plasma clearance, which may in part explain a lower RES dysfunction in animals fed MCT-based lipids (Sobrado et al. 1985). LCT requires carnitine for transport into the mitochondria whereas MCT is not carnitine dependent for $\beta$-oxidation of fatty acids. Hence, MCT may provide a readily available fuel source. A recent investigation in our own laboratory has, however, demonstrated that, carnitine deficiency occurs in less than $4 \%$ of all patients with extreme undernutrition and critical illness (Wennberg et al. 1992). This finding may explain why MCT have not been reported so far to be oxidized more rapidly in applied experiments on stressed or malnourished humans. However, acetyl-L-carnitine can support cellular activities by other means such as providing haemodynamic support (Gasparetto et al. 1991). Future lipids 
may comprise n-6 LCT for essential fatty acids, n-3 LCT for immune modulation and MCT as a readily cleared substrate with minimal effects on the RES system in patients with compromised organ function. Evidence is already available from animal work to suggest that such 'structured lipids' may give metabolic advantages over conventional lipid formulas (Teo et al. 1989).

Evidence that amino acids can serve as more than protein precursors has appeared. Arginine has received increasing interest as an immune stimulator (Daly et al. 1988). The immune effects of arginine may be related to its function as a lymphotropic agent and a stimulator of pituitary growth hormone and insulin. The immuno-enhancing effects of arginine have been illustrated by increased allograft rejection. L-Arginine is also the substrate for nitrous oxide synthesis (Palmer et al. 1988), which may turn out to be an extremely important modulator of vasoactivity in future care of patients with critical illness.

Supported in part by grants from the Swedish Cancer Society (93-B89-22XA, 2014-B8801XA, 2147-B89-04XA), the Medical Research Council (B89-17X-00536-25A, B89-17K08712-01A), Tore Nilson Foundation, Assar Gabrielsson Foundation (AB Volvo), Jubileumskliniken Foundation, Inga-Britt \& Arne Lundberg Research Foundation, Axel \& Margaret Ax:son Johnson Foundation, Swedish and Göteborg Medical Societies and the Medical Faculty, University of Göteborg.

\section{REFERENCES}

Abbott, W. C., Echenique, M. M., Bistrian, B. R., Williams, S. \& Blackburn, G. L. (1983). Nutritional care of the trauma patient. Surgery Gynecology and Obstetrics 157, 585-597.

Alexander, J. W., MacMillan, B. G., Stinnett, J. D., Ogle, C. K., Bozian, R. C., Fischer, J. E., Oakes, J. B., Morris, M. J. \& Krummel, R. (1980). Beneficial effects of aggressive protein feeding in severely burned children. Annals of Surgery 192, 505-517.

Ambrose, N. S., Johnson, M., Burdon, D. W. \& Keighley, M. R. B. (1984). Incidence of pathogenic bacteria from mesenteric lymph nodes and ileal serosa during Crohn's disease surgery. British Journal of Surgery 71, 623-625.

Antonacci, A., Cowles, C. \& Reaves, L. (1984). The role of nutrition in immunologic function. Infection in Surgery 3, 590-597.

Arfvidsson, B., Zachrisson, H., Möller-Loswick, A.-C., Hyltander, A., Sandström, R. \& Lundholm, K. (1991). Effect of systemic hyperinsulinemia on amino acid flux across human legs in postabsorptive state. American Journal of Physiology 260, E46-E52.

Askanazi, J., Carpentier, Y. A., Jeevanandam, M., Michelsen, C. B., Elwyn, D. H. \& Kinney, J. M. (1981). Energy expenditure, nitrogen balance, and norepinephrine excretion after injury. Surgery 89, 478-484.

Bagley, J. S., Wan, J. M. F., Georgieff, M., Forse, R. A. \& Blackburn, G. L. (1991). Cellular nutrition in support of early multiple organ failure. Chest 100, 182S-188S.

Barber, A. E., Jones, W. G., Minei, J. P., Fahey, T. J., Moldawer, L. L., Rayburn, J. L., Fischer, E., Keogh, C. V., Shires, G. T. \& Lowry, S. F. (1990). Harry M. Vars award. Glutamine or fiber supplementation of a defined formula diet: impact on bacterial translocation, tissue composition, and response to endotoxin. JPEN-Journal of Parenteral and Enteral Nutrition 14, 335-343.

Bartlett, R. H., Dechert, R. E., Mault, J. R., Ferguson, S. K., Kaiser, A. M. \& Erlandson, E. E. (1982). Measurement of metabolism in multiple organ failure. Surgery 92, 771-779.

Bartlett, R. H., Mault, J. R., Dechert, R. E., Paimer, J., Swartz, R. D. \& Port, F. K. (1986). Continuous arteriovenous hemofiltration: improved survival in surgical acute renal failure? Surgery 100, 400-408.

Baue, A. E. (1991). Nutrition and metabolism in sepsis and multisystem organ failure. Surgical Clinics of North America 71, 549-565.

Bistrian, B. R., Blackburn, G. L., Vitale, J., Cochran, D. \& Naylor, J. (1976). Prevalence of malnutrition in general medical patients. Journal of the American Medical Association 235, 1567-1570.

Black, P. R., Brooks, D. C., Bessey, P. Q., Wolfe, R. R. \& Wilmore, D. W. (1982). Mechanisms of insulin resistance following injury. Annals of Surgery 196, 420-435.

Border, J. R., Hassett, J, LaDuca, J., Seibel, R., Steinberg, S., Mills, B., Losi, P. \& Border, D. (1987). The gut origin septic states in blunt multiple trauma (ISS $=40$ ) in the ICU. Annals of Surgery 206, 427-448.

Brooks, D. C., Bessey, P. Q., Black, P. R., Aoki, T. T. \& Wilmore, D. W. (1986). Insulin stimulates branched chain amino acid uptake and diminishes nitrogen flux from skeletal muscle of injured patients. Journal of Surgical Research 40, 395-405. 
Burke, D., Alverdy, J. C. \& Aoys, E. (1989). Glutamine supplemented TPN improves gut immune function. Surgical Infection Society Meeting, Denver, CO. p. 12 (abstract).

Cerra, F. B. (1987). The hypermetabolism organ failure complex. World Journal of Surgery 11, 173-181.

Cerra, F. B., McPherson, J. P., Konstantinides, F. N., Konstantinides, N. N. \& Teasley, K. M. (1988). Enteral nutrition does not prevent multiple organ failure syndrome (MOFS) after sepsis. Surgery 104, 727-733.

Cerra, F. B., Mazuski, J., Teasley, K., Nuwer, N., Lysne, J., Shronts, E. \& Konstantinides, F. (1983). Nitrogen retention in critically ill patients is proportional to the branched chain amino acid load. Critical Care Medicine 11, $775-778$.

Cerra, F. B., West, M., Billiar, T. R., Holman, R. T. \& Simmons, R. (1989). Hepatic dysfunction in multiple systems organ failure as a manifestation of altered cell-cell interaction. Second Vienna Shock Forum 563-573.

Chang, R. W. S., Lee, B. \& Jacobs, S. (1989). Identifying ICU patients who would not benefit from total parenteral nutrition. Journal of Parenteral and Enteral Nutrition 13, 535-538.

Clowes, G. H. A., Randall, H. T. \& Cha, C. J. (1980). Amino acid and energy metabolism in septic and traumatized patients. Journal of Parenteral and Enteral Nutrition 4, 195-205.

Dahn, M. S., Langer, P., Lobdell, K., Hans, B., Jacobs, L. A. \& Mitchell, R. A. (1987). Splanchnic and total body oxygen consumption differences in septic and injured patients. Surgery 101, 69-80.

Daly, J. M., Reynolds, J., Thom, A., Kinsley, L., Dietrick-Gallagher, M., Shou, J. \& Ruggieri, B. (1988). Immune and metabolic effects of arginine in the surgical patient. Annals of Surgery 208, 512-523.

Deitch, E. A. (1989). Simple intestinal obstruction causes bacterial translocation in man. Archives of Surgery 124, $699-701$.

Deitch, E. A., Ma, W. J., Ma, L., Berg, R. D. \& Specian, R. D. (1990). Protein malnutrition predisposes to inflammatory-induced gut-origin septic states. Annals of Surgery 211, 560-567.

Detsky, A. S., Baker, J. P., O'Rourke, K. \& Goel, V. (1987). Perioperative parenteral nutrition: a meta-analysis. Annals of Internal Medicine 107, 195-203.

Driks, M. R., Craven, D. E., Celli, B. R., Manning, M., Burke, R. A., Garvin, G. M., Kunches, L. M., Farber, H. W., Wedel, S. A. \& McCabe, W. R. (1987). Nosocomial pneumonia in intubated patients given sucralfate as compared with antacids or histamine type 2 blockers. The role of gastric colonization. New England Journal of Medicine 317, 1376-1382.

Felig, P. (1975). Amino acid metabolism in man. Annual Review of Biochemistry 44, 933-955.

Fine, J., Frank, E. D. \& Ravin, H. A. (1959). The bacterial factor in traumatic shock. New England Journal of Medicine 260, 214-220.

Fong, Y. M., Tracey, K. J, Hesse, D. G., Albert, J. D., Barie, P. S. \& Lowry, S. F. (1990). Influence of enterectomy on peripheral tissue glutamine efflux in critically ill patients. Surgery 107, 321-326.

Friedman, L. M., Furberg, C. D. \& DeMets, D. L. (1981). Sample size. In Fundamentals of Clinical Trials, pp. 69-88. London: J, Wright.

Fry, D. E., Pearlstein, R. L., Fulton, R. L. \& Polk, H. C. (1980). Multiple system organ failure: Role of uncontrolled infection. Archives of Surgery 115, 136-140.

Gasparetto, A.. Corbucci, G. G., De Blasi, R. A., Antonelli, M., Bagiella, E., D'Iddio, S. \& Trevisani, C. (1991). Influence of acetyl-L-carnitine infusion on haemodynamic parameters and survival of circulatory-shock patients. International Joumal of Clinical Pharmacology Research 11, 83-92.

Gould, L., Ettinger, S., Carmichael, A., Lord, P. \& Hofstra, P. (1970). The use of phentolamine in experimental hemorrhagic shock. Angiology 21, 330-335.

Greig, P. D., Elwyn, D. H., Askanazi, J. \& Kinney, J. M. (1987). Parenteral nutrition in septic patients: effect of increasing nitrogen intake. American Journal of Clinical Nutrition 46, 1040-1047.

Hunker, F. D., Bruton, C. W., Hunker, E. M., Durham, R. M. \& Krumdieck, C. L. (1980). Metabolic and nutritional evaluation of patients supported with mechanical ventilation. Critical Care Medicine 8, 628-632.

Hunter, D. C., Jaksic, T., Lewis, D., Benotti, P. N., Blackburn, G. L. \& Bistrian, B. R. (1988). Resting energy expenditure in the critically ill: estimations versus measurement. British Journal of Surgery 75, 875-878.

Hwanu, T. L., O'Dwyer, S. T., Smith, R. J. et al. (1986). Preservation of the small bowel mucosa using glutamine enriched parenteral nutrition. Surgical Forum 37, 56-58.

Jacobs, D. O., Evans, D. A., O'Dwyer, S. T. et al. (1988). Trophic effects of glutamine-enriched parenteral nutrition on colonic mucosa. JPEN-Journal of Parenteral and Enteral Nutrition 12, Suppl. 1, 6.

Jarrett, F., Balish, E., Moylan, J. \& Ellerbe, S. (1978). Clinical experience with prophylactic antibiotic bowel suppression in burn patients. Surgery 83, 523-527.

Jepson, M. M., Bates, P. C., Broadbent, P., Pell, J. M. \& Millward, D. J. (1988). Relationship between glutamine concentration and protein synthesis in rat skeletal muscle. American Journal of Physiology 255, E166-E172.

Jordan, D. A., Miller, C. F., Kubos, K. L. \& Rogers, M. C. (1987). Evaluation of sepsis in a critically ill surgical population. Critical Care Medicine 15, 897-904.

Keene, A. R. \& Cullen, D. J. (1983). Therapeutic intervention scoring system: update. Critical Care Medicine 11, 1.

Knaus, W. A., Zimmerman, J. E., Wagner, D. P. et al. (1984). APACHE-Acute physiologic and chronic health evaluation: a physiologic based classification system. Critical Care Medicine 22, 167.

Ledingham, I. M., Alcock, S. R., Eastaway, A. T., McDonald, J. C., McKay, I. C. \& Ramsay, G. (1988). Triple regimen of selective decontamination of the digestive tract, systemic cefotaxime, and mictobiological surveillance for prevention of acquired infection in intensive care. Lancet $\mathbf{i}, 785-790$. 
Lundholm, K., Bennegård, K., Zachrisson, H., Lundgren, F., Edén, E. \& Möller-Loswick, A.-C. (1987). Transport kinetics of amino acids across the resting human leg. Journal of Clinical Investigation 80, $763-771$.

Lundholm, K., Edström, S., Ekman, L., Karlberg, I., Bylund, A.-C. \& Scherstén, T. (1978). A comparative study of the influence of malignant tumor on host metabolism in mice and man. Evaluation of an experimental model. Cancer 42, 453461.

Lundholm, K., Edström, S., Ekman, L., Karlberg, I., Walker, P. \& Scherstén, T. (1981). Protein degradation in human skeletal muscle tissue: the effect of insulin, leucine, amino acids and ions. Clinical Science 60,319-326.

MacLennan, P. A., Brown, R. A. \& Rennie, M. J. (1987). A positive relationship between protein synthetic rate and intracellular glutamine concentration in perfused rat skeletal muscle. FEBS Letters $215,187-191$.

Marshall, J. C. (1991). The ecology and immunology of the gastrointestinal tract in health and critical illness. Journal of Hospital Infection 19, 7-17.

Marshall, J. C., Christou, N. V., Horn, R. \& Meakins, J. (1987). The microbiology of multiple organ failure: the proximal gastrointestinal tract as an occult reservoir of pathogens. Program, Surgical Infection Society Philadelphia, PA.

Marshall, J. C., Christou, N. V., Horn, R. \& Meakins, J. L. (1988). The microbiology of multiple organ failure. The proximal gastrointestinal tract as an occult reservoir of pathogens. Archives of Surgery 123, 309-315.

Mochizuki, H., Trocki, O., Dominioni, L., Brackett, K. A., Joffe, S. N. \& Alexander, J. W. (1984). Mechanism of prevention of postburn hypermetabolism and catabolism by early enteral feeding. Annals of Surgery 200 , 297-310.

Möller-Loswick, A.-C., Zachrisson, H., Bennegård, K., Sandström, R. \& Lundholm, K. (1991). Insufficient effect of total parenteral nutrition to improve protein balance in peripheral tissues of surgical patients. JPEN-Journal of Parenteral and Enteral Nutrition 15, 669-675.

Moore, E. E. \& Jones, T. N. (1986). Benefits of immediate jejunostomy feeding after major abdominal trauma - a prospective, randomized study. Journal of Trauma 26, 874-881.

Müller, J. M., Brenner, U., Dienst, C. \& Pichlmaier, H. (1982). Preoperative parenteral feeding in patients with gastrointestinal carcinoma. Lancet i, 68-71.

Mutirangura, P., Stonebridge, P. A., Clason, A. E., McClure, J. H., Wildsmith, J. A., Nolan, B., Ruckley, C. V. \& Jenkins, A. McL. (1989). Ten-year review of non-ruptured aortic aneurysms. British Journal of Surgery 76 , $1251-1254$.

Nacht, C. A., Christin, L., Temler, E., Chioléro, R., Jéquier, E. \& Acheson, K. J. (1987). Thermic effect of food: possible implication of parasympathetic nervous system. American Journal of Physiology 253, E481-E488.

Newsholme, E. A. (1990). Metabolic control logic applied to glutamine flux between muscle and the immune system. Clinical Nutrition 9, 113-114.

Offenbartl, K. \& Bengmark, S. (1990). Intraabdominal infections and gut origin sepsis. World Journal of Surgery 14, 19l-195.

Pain, J. A. \& Bailey, M. E. (1986). Experimental and clinical study of lactulose in obstructive jaundice. British Journal of Surgery 73, 775-778.

Palmer, R. M. J., Ashton, D. S. \& Moncada, S. (1988). Vascular endothelial cells synthesize nitric oxide from L-arginine. Nature 333, $664-666$.

Phelps, S. J., Brown, R. O., Helms, R. A., Christensen, M. L., Kudsk, K. \& Cochran, E. B. (1991). Toxicities of parenteral nutrition in the critically ill patient. Critical Care Clinics 7, 725-753.

Pine, R. W., Wertz, M. J., Lennard, E. S., Dellinger, E. P., Carrico, C. J. \& Minshew, B. H. (1983). Determinants of organ malfunction or death in patients with intra-abdominal sepsis: a discriminant analysis. Archives of Surgery 118, 242-249.

Pittiruti, M., Siegel, J. H., Sganga, G., Coleman, B., Wiles, C. E., Belzberg, H., Wedel, S. \& Placko, R. (1985). Increased dependence of leucine in posttraumatic sepsis: leucine/tyrosine clearance ratio as an indicator of hepatic impairment in septic multiple organ failure syndrome. Surgery 98, 378-387.

Pomposelli, J. J., Mascioli, E. A., Bistrian, R. B., Lopes, S. M. \& Blackburn, G. L. (1989). Attenuation of the febrile response in guinea-pigs by fish oil enriched diets. JPEN-Journal of Parenteral and Enteral Nutrition 13, $136-140$.

Quinn, T. \& Askanazi, J. (1987). Nutrition and cardiac disease. Critical Care Clinics 3, 167-184.

Roberts, M. A., Barney, M. H., Trott, M. A., Montez, A. J., Stephens, V. M. \& Lee, D. B. N. (1989). 'Machineless' nutritional hemodialysis. ASAIO Transactions 35, 270-273.

Rothschild, M., Oratz, M. \& Schreiber, S. S. (1975). Regulation of albumin metabolism. Annual Review of Medicine 26, 91-104.

Rush, B. F., Sori, A. J., Murphy, T. F., Smith, S., Flanagan, J. J. \& Machiedo, G. W. (1988), Endotoxemia and bacteremia during hemorrhagic shock. The link between trauma and sepsis? Annals of Surgery 207, $549-554$.

Saba, T. M., Blumenstock, F. A., Scovill, W. A. \& Bernard, H. (1978). Cryoprecipitate reversal of opsonic $\alpha_{2}$-surface binding glycoprotein deficiency in septic surgical and trauma patients. Science 201, 622-624.

Saba, T. M., Dillon, B. C. \& Lanser, M. E. (1983). Fibronectin and phagocytic host defense: relationship to nutritional support. Journal of Parenteral and Enteral Nutrition 7, 62-68.

Sandberg, L. B., Owens, A. J., VanReken, D. E., Horowitz, B., Fredell, J. E., Takyi, Y., Troko, D. M., Horowitz, M. S. \& Hanson, A. P. (1990). Improvement in plasma protein concentrations with fibronectin treatment in severe malnutrition. American Journal of Clinical Nutrition 52, 651-656. 
Sandstedt, S., Lennmarken, C., Symreng, T., Vinnars, E. \& Larsson, J. (1985). The effect of pre-operative total parenteral nutrition on energy-rich phosphates, electrolytes and free amino acids in skeletal muscle of malnourished patients with gastric carcinoma. British Journal of Surgery 72, 920-924.

Sganga, G., Siegel, J. H., Brown, G., Coleman, B., Wiles, C. E., Belzberg, H., Wedel, S. \& Placko, R. (1985). Reprioritization of hepatic plasma protein release in trauma and sepsis. Archives of Surgery 120, $187-199$.

Shaw-Delanty, S. N., Elwyn, D. H., Askanazi, J., Iles, M., Schwarz, Y. \& Kinney, J. M. (1990). Resting energy expenditure in injured, septic, and malnourished adult patients on intravenous diets. Clinical Nutrition $\mathbf{9}$, 305-312.

Sjölin, J., Stjernström, H., Friman, G., Larsson, J. \& Wahren, J. (1990). Total and net muscle protein breakdown in infection determined by amino acid effluxes. American Journal of Physiology 258, E856-E863.

Skeie, B., Kvetan, V., Gil, K. M., Rothkopf, M. M., Newsholme, E. A. \& Askanazi, J. (1990). Branch-chain amino acids: their metabolism and clinical utility. Critical Care Medicine 18, 549-571.

Smith, R. J. \& Wilmore, D. W. (1990). Glutamine nutrition and requirements. JPEN-Journal of Parenteral and Enteral Nutrition 14, Suppl. 4, 94S-99S.

Sobrado, J., Moldawer, L. L., Pomposelli, J. J., Mascioli, E. A., Babayan, V. K., Bistrian, B. R. \& Blackburn, G. L. (1985). Lipid emulsions and reticuloendothelial system function in healthy and burned guinea pigs. American Journal of Clinical Nutrition 42, 855-863.

Souba, W. W., Klimberg, V. S., Hautamaki, R. D., Mendenhall, W. H., Bova, F. C., Howard, R. J., Bland, K. I. \& Copeland, E. M. (1990). Oral glutamine reduces bacterial translocation following abdominal radiation. Journal of Surgical Research 48, 1-5.

Souba, W. W., Smith, R. J. \& Wilmore, D. W. (1985). Glutamine metabolism by the intestinal tract. JPEN-Journal of Parenteral and Enteral Nutrition 9, 608-617.

Stehle, P., Zander, J., Mertes, N., Albers, S., Puchstein, C. H., Lawin, P. \& Fürst, P. (1989). Effect of parenteral glutamine peptide supplements on muscle glutamine loss and nitrogen balance after major surgery. Lancet $\mathbf{i}$, 231-233.

Steinmetz, O. K. \& Meakins, J. L. (1991). Care of the gut in the surgical intensive care unit: fact or fashion? Canadian Journal of Surgery 34, 207-215.

Sturm, H. A., Lewis, F. R., Trentz, O. et al. (1979). Cardiopulmonary parameters and prognosis after severe multiple trauma. Journal of Trauma 19, 305-318.

Tancrede, C. H. \& Andremont, A. O. (1985). Bacterial translocation and gram negative bacteremia in patients with hematological malignancies. Journal of Infectious Diseases 152, 99-103.

Teo, T. C., Demichele, S. J., Selleck, K. M., Babayan, V. K., Blackburn, G. L. \& Bistrian, B. R. (1989). Administration of structured lipid composed of MCT and fish oil reduces net protein catabolism in enterally fed burned rats. Annals of Surgery 210, 100-107.

Ternell, M., Edström, S. \& Lundholm, K. (1983). Transcriptional and translational activity in relation to oxygen consumption in isolated liver cells from sarcoma-bearing mice. Cancer Biochemistry and Biophvsics 6, $213-219$.

Ternell, M., Zachrisson, H. \& Lundholm, K. (1988). RNA polymerase activity and protein synthesis in mouse tumor-host liver compared to benign para-neoplastic reactions. International Journal of Cancer 42, 464-469.

Trocki, O., Mochizuki, H., Dominioni, L. \& Alexander, J. W. (1986). Intact protein versus free amino acids in the nutritional support of thermally injured animals. JPEN-Journal of Parenteral and Enteral Nutrition 10 , $139-145$

VA Total Parenteral Nutrition Cooperative Study Group (1991). Perioperative total parenteral nutrition in surgical patients. New England Journal of Medicine 325, 525-532.

Van Saene, H. F. K., Stoutenbeek, C. P., Miranda, D. R. \& Zandstra, D. F. (1983). A novel approach to infection control in the intensive care unit. Acta Anaesthesiologica Belgica 34, 193-208.

Vincent, P., Colombel, J. F.\& Lescut, D. (1988). Bacterial translocation in patients with colorectal cancer. Journal of Infectious Diseases 158, 1395-1396.

von Meyenfeldt, M. F., Soeters, P. B., Vente, J. P., van Berlo, C. L., Rouflart, M. M., de Jong, K. P., van der Linden, C. J. \& Gouma, D. J. (1990). Effect of branched chain amino acid enrichment of total parenteral nutrition on nitrogen sparing and clinical outcome of sepsis and trauma : a prospective randomized double blind trial. British Journal of Surgery 77, 924-929.

Wan, J. M., \& Grimble, R. F. (1987). Effect of dietary linoleate content on the metabolic response of rats to Escherichia coli endotoxin. Clinical Science 72, 383-385.

Weiss, L., Danielson, B. G., Wikström, B., Hedstrand, U. \& Wahlberg, J. (1989). Continuous arteriovenous hemofiltration in the treatment of 100 critically ill patients with acute renal failure: report on clinical outcome and nutritional aspects. Clinical Nephrology 31, 184-189.

Wennberg, A., Hyltander, A., Sjöberg, Å., Arfvidsson, B., Sandström, R., Wickström, I. \& Lundholm, K. (1992), , Prevalence of carnitine depletion in critically ill patients with undernutrition. Metabolism 41, 165-171.

Wusteman, M. \& Elia, M. (1991). Effect of glutamine infusions on glutamine concentration and protein synthetic rate in rat muscle. JPEN-Journal of Parenteral and Enteral Nutrition 15, 521-525.

Xian-jun, M., Tao, Y., Ping, Z. \& Ping, W. (1988). Experimental study on the effects of adrenergic blockade to multiple organ failure. Chinese Medical Journal 101, 209-212. 